\title{
Prevalence of the insulin resistance syndrome in obesity
}

\author{
R M Viner, T Y Segal, E Lichtarowicz-Krynska, P Hindmarsh
}

Arch Dis Child 2005;90:10-14. doi: 10.1136/adc.2003.036467

See end of article for authors' affiliations ...................

Correspondence to: Dr R Viner, Department of Paediatrics, Middlesex Hospital, Mortimer St, London WIT 3AA, UK; R.Viner@ich.ucl.ac.uk

Accepted 12 October 2003

\begin{abstract}
Aims: To assess prevalence of the insulin resistance syndrome (IRS: obesity, abnormal glucose homoeostasis, dyslipidaemia, and hypertension) in obese UK children and adolescents of different ethnicities and to assess whether fasting data is sufficient to identify IRS in childhood obesity.

Methods: A total of 103 obese (BMI >95th centile) children and adolescents 2-18 years of age referred for assessment underwent an oral glucose tolerance test, measurement of fasting lipids, and blood pressure determination. Main outcome measures were prevalence of components of IRS by modified WHO criteria, with IRS defined as $\geqslant 3$ components (including obesity).

Results: There were 67 girls $(65 \%)$. BMl $z$-score ranged from 1.65 to 6.15 , with $72 \%$ having a $z$-score $\geqslant 3.0$. Abnormal glucose homoeostasis was identified in $46 \%$ (hyperinsulinism in $40 \%$, impaired fasting glucose in $0.8 \%$, impaired glucose tolerance in $11 \%$ ). No subjects had silent type 2 diabetes. Dyslipidaemia was identified in $30 \%$ and hypertension in $32 \%$. Thirty one per cent had obesity alone, $36 \%$ had two components, $28 \%$ had three, and $5 \%$ had all four components. Birth weight, BMI, and family history of IRS were not associated with risk of IRS. Higher age increased the risk of IRS; however the syndrome was seen in $30 \%$ of children under 12 years. The use of fasting glucose and insulin data for identifying IRS had a sensitivity of $88 \%$ and specificity of $100 \%$.

Conclusions: One third of obese children and adolescents have the IRS; however type 2 diabetes is rare. Obese children with the IRS may form a high risk group to whom scarce intervention resources should be targeted. Further work is needed to develop appropriate screening programmes for IRS components in significantly obese children.
\end{abstract}

O besity in childhood and adolescence has been shown to increase the risk of the insulin resistance syndrome $(\text { IRS })^{1}$ and cardiovascular mortality in adult life. ${ }^{2}$ The clustering of major IRS components, including obesity, hypertension, dyslipidaemia, and atherosclerosis, has been shown in children and adolescents ${ }^{3}{ }^{4}$ and tracks strongly into adult life. ${ }^{5}$ This tracking has been shown to increase with increasing age and obesity. ${ }^{5}$ Many studies have shown that individual IRS components such as insulin resistance, dyslipidaemia, and hypertension are highly inter-correlated and significantly more common in obese children than in those of normal weight. ${ }^{6-9}$ However the prevalence of IRS in obese children and adolescents has been poorly documented. Epidemiological data from the USA suggest that the clustering of obesity with two or more of hyperinsulinaemia, dyslipidaemia, and hypertension is seen in $20 \%$ of obese children and adolescents. ${ }^{8}$ However, this data is drawn from a historical cohort of US white and black children, did not use modern more restrictive definitions of obesity, ${ }^{10}$ and did not use a standardised definition of IRS (for example, the WHO definition). ${ }^{11}$ The prevalence of the syndrome in contemporary obese UK children of different ethnicities is not documented.

Identification of obese children with additional IRS components may define a high risk group within the obese child population. There is little consensus on which obese children require treatment, as available guidelines focus on assessment in primary care ${ }^{12}$ and there are no accepted definitions of extreme or morbid obesity in childhood. Given the scale of the problem, the paucity of effective interventions, ${ }^{13}$ and the lack of available clinical resources, there is an urgent need to identify high risk groups who require treatment within the obese child population. Additionally, little guidance is available for clinicians on the appropriate investigations required for children and adolescents with obesity. Guidance has been published on screening for lipids and blood pressure, ${ }^{14}$ but with little information available on screening for glucose and insulin abnormalities, particularly as to whether fasting samples alone or an oral glucose tolerance test (OGTT) are required to identify abnormal glucose homoeostasis. In adults, there is increasing evidence that measurements of fasting glucose do not pick up many subjects with impaired glucose tolerance, ${ }^{15}$ particularly in certain ethnicities. ${ }^{16}$

We assessed the prevalence of the major components of IRS in a clinical sample of obese UK children and adolescents of different ethnicities using two definitions of obesity. We then examined whether demographic, clinical, and laboratory factors were associated with increased risk of IRS. Finally we explored whether fasting insulin and glucose are sufficient to screen for abnormalities in glucose homoeostasis in childhood obesity.

\section{METHODS}

Subjects were children and adolescents 2-18 years of age who underwent assessment of obesity in our joint paediatric and adolescent endocrine service between 1999 and 2002 . Subjects were referred by their GP or local paediatrician for medical assessment of obesity. Subjects assessed prior to June 2002 were identified through a retrospective examination of hospital records. Subjects from July 2002 onwards had data collected prospectively. Only those who had had secondary or known genetic causes of obesity excluded were included in this analysis. Obesity was defined in two ways: firstly, as body mass index (BMI) $\geqslant 95$ th centile for age and sex according to the UK 1990 growth reference; ${ }^{17}$ secondly, we defined extreme obesity as BMI z score $\geqslant 3.0(\geqslant 99.86$ th centile) as there are no standard definitions of extreme obesity in childhood.

Abbreviations: BMI, body mass index; IRS, insulin resistance syndrome; OGT, oral glucose tolerance test 
Subjects were assessed after a 12 hour overnight fast. Subjects underwent an OGTT (1.75 mg glucose $/ \mathrm{kg}$ ) with plasma insulin and blood glucose concentrations measured at $0,30,60$, and 120 minutes. Plasma insulin was estimated by the Abbott AxSYM method. Fasting serum lipid concentrations (total cholesterol and triglycerides) were measured. Clinical policy meant that HDL cholesterol was measured in all adolescents but only in those children with abnormal total cholesterol or triglycerides. Blood pressure was measured in the recumbent position using an oscillometric method with an appropriate sized cuff for arm diameter. Data are reported as the average of two consecutive readings. Birth weight, ethnicity, pubertal status, presence of acanthosis nigricans, and family history of components of IRS were recorded or obtained from hospital records.

Abnormal glucose homoeostasis and IRS were defined following modified WHO criteria ${ }^{11}$ adapted for children (table 1). Hyperinsulinism was defined from norms for pubertal stage: ${ }^{18}$ prepubertal $\geqslant 15 \mathrm{mU} / \mathrm{l}$, mid-puberty (stages $2-4) \geqslant 30 \mathrm{mU} / \mathrm{l}$. Post-pubertal hyperinsulinism was defined as per adult WHO criteria $(\geqslant 20 \mathrm{mU} / \mathrm{l}) .{ }^{11}$ Hypertension was defined as systolic blood pressure $\geqslant 95$ th centile for age and sex. ${ }^{19}$ Abnormal fasting lipids were defined from normative data. $^{20}$ IRS was defined as having three or more IRS components. ${ }^{11}$

Insulin sensitivity from fasting samples was estimated using the Homeostasis Model Assessment (HOMA-IR), ${ }^{21}$ the Quantitative Insulin Check Index (QUICKI index), ${ }^{22}$ and fasting insulin:glucose ratio (FIGR). ${ }^{23}$ HOMA-IR was calculated as fasting insulin $(\mathrm{U} / \mathrm{l}) \times$ fasting glucose $(\mathrm{mmol} / \mathrm{l}) / 22.5$ (higher values indicate greater insulin resistance). QUICKI was calculated as $1 /(\log$ fasting insulin $(U / 1)+\log$ fasting glucose $(\mathrm{mg} / \mathrm{dl})$ ) (higher QUICKI values indicate greater insulin sensitivity). FIGR was calculated as fasting insulin $(\mathrm{U} / \mathrm{l}) /$ fasting glucose $(\mathrm{mg} / \mathrm{dl})$. The homoeostasis model assessment was used to estimate pancreatic $\beta$ cell function (HOMA-B\%) and was calculated as $20 \times$ fasting insulin (U/l)/ (fasting glucose $(\mathrm{mmol} / \mathrm{l})-3.5) \cdot{ }^{21}$

\section{Analysis}

The $\chi^{2}$ test or ANOVA with Bonferroni post-hoc test were used to assess differences between groups where appropriate. The diagnostic yield of using only fasting insulin and glucose values compared to full OGTT data was examined by sensitivity analysis. Factors associated with risk of IRS were examined by logistic regression. Data were analysed using SPSS 10.

\section{RESULTS}

A total of 103 children and adolescents aged 2.3-18 years underwent assessment (36 males, 35\%). Mean age was 11.9 years (SE 0.4) (males 12.9 (SE 0.6) years; females 11.2 (SE

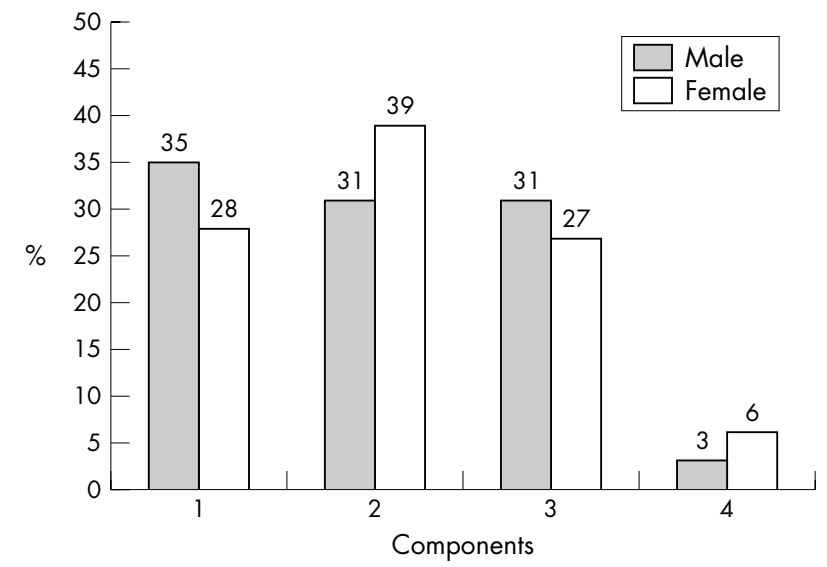

Figure 1 Number of IRS components by sex (including obesity).

$0.5)$ years). BMI z-score ranged from 1.65 to 6.15 (mean 3.48), and all but five subjects had BMI z-score $>2.35$ ( $>99$ th centile). Seventy two per cent were extremely obese (BMI $z$-score $\geqslant 3.0)$. There was no sex difference in mean BMI zscore or extreme obesity. Thirty nine $(38 \%)$ were prepubertal, 27 (26\%) were in mid-puberty (breast, genital stages 2-4), and $37(36 \%)$ were post-pubertal. Acanthosis nigricans was noted in $45 / 82$ subjects (55\%). Data on ethnicity was available for 93 subjects (90\%): white 54 (58\%), black 8 (9\%), south Asian 27 (29\%), and other or mixed ethnicity 4 (4\%). Fifty subjects (49\%) reported a family history of any IRS component in the first or second generation. Mean birth weight was $3.51 \mathrm{~kg}$ (SD 0.82, range $1.45-5.56 \mathrm{~kg}$ ).

Table 2 shows the prevalence of individual components of IRS by sex. Impaired fasting glucose was identified in only one subject $(0.8 \%)$, impaired glucose tolerance in $11(11 \%)$, and no subjects had silent diabetes. Hyperinsulinism was identified in $41(40 \%)$ subjects. Overall, abnormal glucose homoeostasis was identified in $46(45 \%)$ and was not associated with sex, ethnicity, or pubertal status. High total serum cholesterol concentrations were noted in $18(18 \%)$, high serum triglyceride concentrations in $20 \%$, and low HDL in $4(10 \%)$ of the $40(39 \%)$ subjects who had HDL measured. Overall, dyslipidaemia was identified in 31 (30\%) with no significant differences by gender, pubertal status, or ethnicity. Mean systolic blood pressure z-score was 1.25 (SE 0.13; range -1.34 to 3.36$)$. Hypertension was noted in 33 (32\%) with no significant differences by sex or pubertal status. Hypertension was significantly more common in white young people $(47 \%)$ than black ( $14 \%)$ or Asian $(20 \%)$ young people $\left(\chi^{2}=8.1, \mathrm{p}<0.05\right)$

Table 1 Definition of components of the IRS"

\begin{tabular}{|c|c|}
\hline IRS component & Definition \\
\hline 1. Obesity & $\mathrm{BMI} \geqslant 95$ th centile for age and sex \\
\hline 2. Abnormal glucose homoeostasis & $\begin{array}{l}\text { Any of the following: } \\
\text { A. Fasting hyperinsulinaemia } \\
\text { B. Impaired fasting glucose ( } \geqslant 6.1 \mathrm{mM} / \mathrm{l}) \\
\text { C. Impaired glucose tolerance: glucose at } 120 \mathrm{~min} \geqslant 7.8 \mathrm{mM} / \mathrm{I}\end{array}$ \\
\hline 3. Hypertension & Systolic blood pressure $\geqslant 95$ th centile for age and sex \\
\hline 4. Dyslipidaemia & $\begin{array}{l}\text { Any of the following: } \\
\text { A. High triglycerides }(\geqslant 1.75 \mathrm{mM} / \mathrm{l}) \\
\text { B. Low } \mathrm{HDL}(<0.9 \mathrm{mM} / \mathrm{l}) \\
\text { C. High total cholesterol ( } \geqslant 95 \text { th centile) }\end{array}$ \\
\hline
\end{tabular}




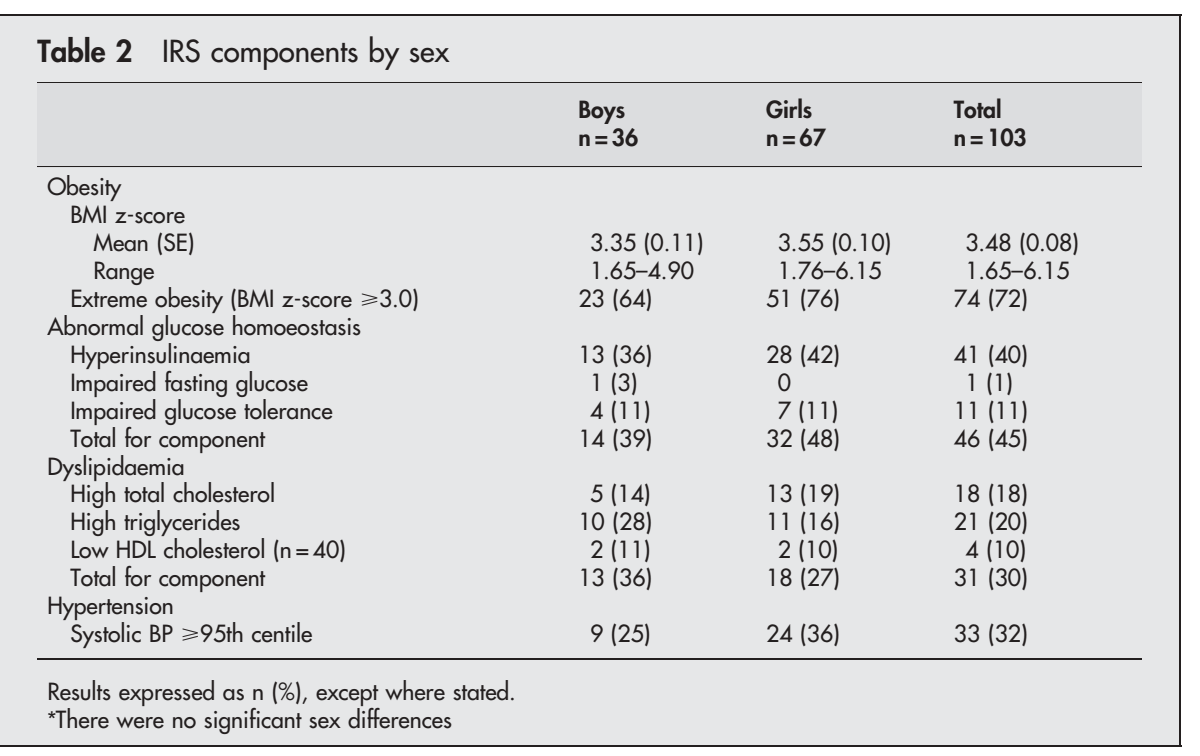

The prevalence of IRS components by sex using the definitions in table 1 is shown in fig 1 . Thirty two subjects (31\%) had obesity alone (one component). Two components were identified in 37 (36\%), three components in $29(28 \%)$, and all four components in five $(5 \%)$. IRS, defined as $\geqslant 3$ components, was found in 34 (33\%), with a non-significantly higher rate among adolescents aged $12-18$ years (36\%) than among children aged $2-11$ years $(30 \%)$. There were no significant differences in the prevalence of IRS by sex or pubertal status. There was a trend for IRS to be more common among white $(37 \%)$ than black (13\%) or Asian ( $22 \%)$ young people $\left(\chi^{2}=6.6, p=0.08\right)$. While IRS was slightly more common among the extremely obese $(35 \%)$ compared with the simply obese $(28 \%)$ (z-score $<3.0)$, this difference was not significant.

Logistic regression showed that the only clinical or demographic factor associated with risk of IRS was higher age (OR controlled for pubertal status $1.13, \mathrm{p}=0.04,95 \%$ CI 1.09 to 2.60). Birth weight, BMI and BMI z-score, the presence of acanthosis nigricans, and family history of IRS were not associated with risk of IRS.

We assessed the usefulness of fasting insulin and glucose values for assessing IRS by comparing the prevalence of IRS using only fasting information to define abnormal glucose homoeostasis (either fasting hyperinsulinaemia or impaired fasting glucose) with the prevalence previously derived from full OGTT data. Using the fasting definition, 33 (32\%) had one component, 40 (39\%) two components, 25 (24\%) three components, and five $(5 \%)$ had all four components. The prevalence of the full syndrome ( $\geqslant 3$ components) was $29 \%$ using this "fasting" definition.

Table 3 shows the diagnostic power of using only fasting insulin and glucose for IRS. Sensitivity analysis for the relations between the fasting definition of IRS and the OGTT definition (taken as "actual disease") showed the following: specificity, $69 / 69=100 \%$; sensitivity, $30 / 34=88 \%$; positive predictive value, $30 / 30=100 \%$; negative predictive value, $69 /$ $73=95 \%$.

We further investigated whether using calculated fasting indices of insulin sensitivity or secretion improved the diagnostic yield of fasting samples, particularly in terms of identifying patients with impaired glucose tolerance but not either fasting hyperinsulinaemia or impaired fasting glucose. Of the 11 subjects with impaired glucose tolerance, four had normal fasting insulin and glucose and did not have abnormal glucose homoeostasis by the "fasting" definition.
However the same four subjects also had values for HOMAIR, HOMA-B\%, FIGR, and QUICKI within I SD of means from normal weight children. ${ }^{24}$ In multivariate analysis controlling for age, sex, and pubertal status, fasting glucose, insulin, HOMA-IR, QUICKI, and FIGR were not predictive of impaired glucose tolerance.

\section{DISCUSSION}

We found $33 \%$ of obese children and adolescents of different ethnicities to have evidence of IRS as defined by $\geqslant 3$ of obesity, abnormal glucose homoeostasis, dyslipidaemia, and hypertension. A further third of our sample had one additional IRS component in addition to obesity. As epidemiological evidence suggests that the clustering of IRS components tracks strongly into adult life and greatly increases cardiovascular risk, ${ }^{525}$ these observations in children raise the possibility that obese children with two or more additional IRS components might form a high risk group within the obese child population.

In contrast to concerns about the rising incidence of type 2 diabetes in obese children and adolescents, ${ }^{26}{ }^{27}$ we found that none of our obese children or adolescents of any ethnicity had silent type 2 diabetes. This finding is similar to that of a recent US report which found silent type 2 diabetes in only $3 \%$ of very obese subjects 4-18 years of age. ${ }^{28}$ We also found low rates of pre-diabetic glucose abnormalities, with $11 \%$ having impaired glucose tolerance and $1 \%$ having impaired fasting glucose. These rates are lower than estimates of impaired glucose tolerance in the aforementioned US clinical sample $(20-25 \%)^{28}$ but higher than those from an Italian clinical sample $(4.5 \%){ }^{6}$ It is likely that these differences reflect the differing clinical and ethnic populations studied. Our findings of a high prevalence of IRS but a low prevalence of glucose intolerance and type 2 diabetes reflect the range of abnormalities of glucose homoeostasis associated with obesity in childhood. While increasing obesity may over time unmask type 2 diabetes in those genetically predisposed to $\beta$ cell failure, insulin insensitivity is the earlier and predominant abnormality of glucose homoeostasis found in obesity. ${ }^{29}$

One aim of this study was to identify factors that clinicians could use to guide decisions on the medical investigation of obese children. We were unable to identify clinical factors that identified obese children at greater risk of IRS. While age increased IRS risk, the youngest subject with IRS was 4.0 years and IRS was seen in 30\% of children under 12 years, suggesting that age based cut-offs are not useful in 
Table 3 Diagnostic power of fasting compared with OGT definitions of IRS

\begin{tabular}{llrr}
\hline & \multicolumn{2}{l}{ Defined using OGT data } \\
\cline { 3 - 4 } \cline { 3 - 3 } & & IRS & $<3$ components \\
\hline \multirow{2}{*}{ Defined using "fasting" data } & IRS & 30 & 0 \\
& $<3$ components & 4 & 69 \\
\hline
\end{tabular}

identifying those who require investigation. The lack of relation between BMI (either raw or z-score) and IRS almost certainly reflects the constricted range of BMI among the subjects, but also suggests that that higher BMI thresholds (for example, $\mathrm{z}$-score $\geqslant 3.0$ ) are not clinically useful in defining a high risk group. We were surprised that neither ethnicity nor family history of IRS were related to risk of IRS, particularly given evidence of heritability of IRS components ${ }^{30}$ and variation in insulin sensitivity between children of different ethnicities. ${ }^{31-33}$ This undoubtedly relates in part to the size of the sample studied but suggests that family and ethnic background cannot be used in clinical practice to select obese children and adolescents for further investigations.

Our findings relate to obesity defined as BMI $\geqslant 95$ th centile; however, all but five subjects ( $5 \%$ ) were obese by the more restrictive newer International Obesity Task Force definitions, which relate to an adult $\mathrm{BMI} \geqslant 30 \mathrm{~kg} / \mathrm{m}^{2} .{ }^{10}$ Of these five subjects, three had one IRS component, one had two components, and one had all four components. There was no significant difference in the prevalence of IRS when obesity was defined by either BMI centile or the international definition.

We also studied which measurements of glucose and insulin were most useful in screening for abnormalities in glucose homoeostasis associated with IRS. Insulin and glucose data from the OGTT identified abnormalities in $45 \%$ of subjects. In contrast, measurement of fasting blood glucose alone would have identified only one subject (1\%) and measurement of blood glucose alone during an OGTT would have identified only 12 subjects. Thus screening for abnormalities in glucose homoeostasis in obese children using blood glucose alone, either fasting or during an OGTT, is likely to have extremely low yield and measurements of insulin and glucose are needed to identify the most prevalent abnormalities in glucose homoeostasis. Identification of all the abnormalities in glucose homoeostasis requires the subject to undergo an OGTT measuring glucose and insulin, as fasting insulin and glucose are thought to predominantly reflect insulin secretion and hepatic insulin sensitivity, whereas data from the later stages of the OGTT are thought to reflect peripheral as well as hepatic insulin sensitivity. ${ }^{34} 15$ However, we found that in our sample of obese children and adolescents, the use of raw fasting insulin and glucose data alone had high specificity and an acceptable sensitivity of $88 \%$ in identifying subjects with IRS, with only $4 \%$ of subjects with the syndrome missed if fasting values alone were used. We did not find that indices of insulin sensitivity (HOMA-IR, QUICKI, FIGR) or secretion (HOMA-B\%) calculated from fasting values were useful in predicting risk of IRS over and above the fasting values themselves.

\section{Limitations of these data}

Data from clinical samples may not be representative of the general population and selection and referral bias may have inflated our estimate of the prevalence of IRS. However, this was a regional referral sample representing a population of major concern to clinicians and included significant numbers of children from white, Asian, and black ethnicities. Additionally, no subjects had been referred because of previously identified abnormalities of glucose homoeostasis, and hypertension had been previously identified in less than $5 \%$ of the sample. Our modifications of the standard adult IRS criteria may have affected our results. Our definition of hyperinsulinism by pubertal status produced a more conservative estimate of IRS prevalence than using the standard adult definition (data not shown). We used high total cholesterol in addition to high triglycerides and low HDL to define dyslipidaemia, as evidence suggests that high total cholesterol remains the best marker for cardiovascular risk during childhood and adolescence. ${ }^{35}$

\section{Conclusions}

Clinical concerns over obesity in childhood and adolescence should focus on the presence of additional IRS components, including abnormalities of glucose homoeostasis, hypertension, and dyslipidaemia. We suggest that clinicians should consider screening all significantly obese children and adolescents regardless of age, ethnicity, or pubertal status for IRS. Fasting measures have acceptable sensitivity and specificity if fasting insulin is measured as well as glucose.

Those with three or more IRS components may form an appropriate group for intervention, which may include medical therapy for hyperinsulinaemia, ${ }^{36}$ dyslipidaemia, or hypertension as well as multidisciplinary weight control programmes. The value of such interventions needs to be assessed by formal clinical trials with long term follow up of outcome.

\section{Authors' affiliations}

R M Viner, T Y Segal, E Lichtarowicz-Krynska, P Hindmarsh, London Centre for Paediatric Endocrinology \& Diabetes, Royal Free and University College Medical School, University College London, UK

\section{REFERENCES}

1 Reaven GM. Banting lecture 1988. Role of insulin resistance in human disease. Diabetes 1988;37:1595-607.

2 Srinivasan SR, Myers L, Berenson GS. Predictability of childhood adiposity and insulin for developing insulin resistance syndrome (syndrome $\mathrm{X}$ ) in young adulthood: the Bogalusa Heart Study. Diabetes 2002;51:204-9.

3 Freedman DS, Dietz WH, Srinivasan SR, et al. The relation of overweight to cardiovascular risk factors among children and adolescents: the Bogalusa Heart Study. Pediatrics 1999;103:1175-82.

4 Young-Hyman D, Schlundt DG, Herman L, et al. Evaluation of the insulin resistance syndrome in 5- to 10-year-old overweight/obese African-American children. Diabetes Care 2001;24:1359-64.

5 Bao W, Srinivasan SR, Wattigney WA, et al. Persistence of multiple cardiovascular risk clustering related to syndrome $X$ from childhood to young adulthood. The Bogalusa Heart Study. Arch Intern Med 1994;154:1842-7.

6 Invitti C, Guzzaloni G, Gilardini L, et al. Prevalence and concomitants of glucose intolerance in European obese children and adolescents. Diabetes Care 2003;26:118-24.

7 Morrison JA, Barton BA, Biro FM, et al. Overweight, fat patterning, and cardiovascular disease risk factors in black and white boys. J Pediatr 1999; 135:451-7.

8 Freedman DS, Khan LK, Dietz WH, et al. Relationship of childhood obesity to coronary heart disease risk factors in adulthood: the Bogalusa Heart Study. Pediatrics 2001; 108:712-18

9 Sinaiko AR, Jacobs DR Jr, Steinberger J, et al. Insulin resistance syndrome in childhood: associations of the euglycemic insulin clamp and fasting insulin with fatness and other risk factors. J Pediatr 2001;139:700-7.

10 Cole TJ, Bellizzi MC, Flegal KM, et al. Establishing a standard definition for child overweight and obesity worldwide: international survey. BMJ 2000;320:1240-3. 
11 Alberti KG, Zimmet PZ. Definition, diagnosis and classification of diabetes mellitus and its complications. Part 1: diagnosis and classification of diabetes mellitus provisional report of a WHO consultation. Diabet Med 1998; 15:539-53.

12 Gibson P, Edmunds L, Haslam DW, et al. An approach to weight management in children and adolescents (2-18 years) in primary care. London: Royal College of Paediatrics, 2002.

13 Reilly JJ, Wilson ML, Summerbell CD, et al. Obesity: diagnosis, prevention, and treatment; evidence based answers to common questions. Arch Dis Child 2002;86:392-4.

14 Williams CL, Hayman LL, Daniels SR, et al. Cardiovascular health in childhood: a statement for health professionals from the Committee on Atherosclerosis, Hypertension, and Obesity in the Young (AHOY) of the Council on Cardiovascular Disease in the Young, American Heart Association. Circulation 2002;106:143-60.

15 Carnevale Schianca GP, Rossi A, Sainaghi PP, et al. The significance of impaired fasting glucose versus impaired glucose tolerance: importance of insulin secretion and resistance. Diabetes Care 2003;26:1333-7.

16 Anand SS, Razak F, Vuksan V, et al. Diagnostic strategies to detect glucose intolerance in a multiethnic population. Diabetes Care 2003;26:290-6.

17 Cole TJ, Freeman JV, Preece MA. Body mass index reference curves for the UK, 1990. Arch Dis Child 1995;73:25-9.

18 Goran MI, Gower BA. Longitudinal study on pubertal insulin resistance. Diabetes 2001;50:2444-50.

19 Task Force on Blood Pressure Control in Children. Report of the Second Task Force on Blood Pressure Control in children-1987. Pediatrics 1987:79:1-25

20 Hickman TB, Briefel RR, Carroll MD, et al. Distributions and trends of serum lipid levels among United States children and adolescents ages 4-19 years: data from the Third National Health and Nutrition Examination Survey. Prev Med 1998;27:879-90.

21 Matthews DR, Hosker JP, Rudenski AS, et al. Homeostasis model assessment: insulin resistance and beta-cell function from fasting plasma glucose and insulin concentrations in man. Diabetologia 1985;28:412-19.

22 Katz A, Nambi SS, Mather K, et al. Quantitative insulin sensitivity check index a simple, accurate method for assessing insulin sensitivity in humans. J Clin Endocrinol Metab 2000;85:2402-10.

23 Guerrero-Romero F, Rodriguez-Moran M. Glucose intolerance is predicted by the high fasting insulin-to-glucose ratio. Diabetes Metab 2001;27:117-21.
24 Uwaifo GI, Fallon EM, Chin J, et al. Indices of insulin action, disposal and secretion derived from fasting samples and clamps in normal glucose-tolerant black and white children. Diabetes Care 2002;25:2081-7.

25 Chen W, Srinivasan SR, Elkasabany A, et al. Cardiovascular risk factors clustering features of insulin resistance syndrome (syndrome $X$ ) in a biracial (black-white) population of children, adolescents, and young adults: the Bogalusa Heart Study. Am J Epidemiol 1999;150:667-74.

26 Fagot-Campagna A, Pettitt DJ, Engelgau MM, et al. Type 2 diabetes among North American children and adolescents: an epidemiologic review and a public health perspective. J Pediatr 2000;136:664-72.

27 Drake AJ, Smith A, Betts PR, et al. Type 2 diabetes in obese white children. Arch Dis Child 2002;86:207-8.

28 Sinha R, Fisch G, Teague B, et al. Prevalence of impaired glucose tolerance among children and adolescents with marked obesity. N Engl J Med 2002;346:802-10

29 Gerich JE. Contributions of insulin-resistance and insulin-secretory defects to the pathogenesis of type 2 diabetes mellitus. Mayo Clin Proc 2003;78:447-56

30 Freeman MS, Mansfield MW, Barrett JH, et al. Heritability of features of the insulin resistance syndrome in a community-based study of healthy families. Diabet Med 2002;19:994-9.

31 Whincup PH, Gilg JA, Papacosta $O$, et al. Early evidence of ethnic differences in cardiovascular risk: cross sectional comparison of British South Asian and white children. BMJ 2002;324:635.

32 Goran MI, Bergman RN, Cruz ML, et al. Insulin resistance and associated compensatory responses in African-American and Hispanic children. Diabetes Care 2002;25:2184-90.

33 Tortolero SR, Goff DC Jr, Nichaman MZ, et al. Cardiovascular risk factors in Mexican-American and non-Hispanic white children: The Corpus Christi Child Heart Study. Circulation 1997;96:418-23.

34 Matsuda M, DeFronzo RA. Insulin sensitivity indices obtained from oral glucose tolerance testing: comparison with the euglycemic insulin clamp. Diabetes Care 1999;22:1462-70.

35 Berenson GS, Srinivasan SR. Consideration of serum cholesterol in risk factor profiling for all young individuals. Nutr Metab Cardiovasc Dis 2001;11(suppl 5):1-9.

36 Decsi T, Molnar D. Insulin resistance syndrome in children: pathophysiology and potential management strategies. Paediatr Drugs 2003;5:291-9.

\section{ARCHIVIST}

\section{Environment, injury, and child health in Europe}

$\mathrm{T}$ he children's environment and health action plan for Europe (CEHAPE) was on the agenda for the Fourth Ministerial Conference on Environment and Health held in

Budapest at the end of June 2004. An action plan released by the European Commission on June 9 included 13 actions to be implemented by 2010, including expansion of environmental research and training. A WHO sponsored study of the environmental burden of disease (EBD) in children (Francesca Valent and colleagues. Lancet 2004;363:2032-9) was carried out in order to provide data for the development of CEHAPE.

The EBD was assessed in terms of deaths and disability-adjusted life years (DALYs) from published data but gaps in data and potential sources of error and uncertainty have limited the analyses. Nevertheless useful conclusions can be drawn. Analyses were restricted to four environmental factors (outdoor air pollution, indoor air pollution, water and sanitation, and lead exposure) and injury. It was estimated that outdoor air pollution accounted for $6.4 \%$ of all deaths in children under the age of 5 years and acute lower respiratory tract infection due to indoor air pollution accounted for $4.6 \%$ of deaths in this age group. Diarrhoea from inadequate water supply and sanitation accounted for $5.3 \%$ of deaths in children under the age of 15 years. Morbidity was not estimated for outdoor air pollution but it was calculated that indoor air pollution accounted for $3.1 \%$ of DALYs (children aged $0-4$ years), lead exposure (by causing mild mental retardation) for $4.4 \%$ of DALYs (children aged $0-4$ ), and inadequate water and sanitation for 3.5\% of DALYs in children aged 0-14 years. (Figures for deaths and DALYs from indoor air pollution are not included for western Europe because of lack of adequate data.) Injury was the cause of $22.6 \%$ of deaths and $19 \%$ of DALYs in children and adolescents aged $0-19$ years.

The WHO divides Europe into three subregions: EurA (broadly western Europe), EurB (a swathe of countries from Poland, through the Balkans and Turkey, to the southern countries of the former USSR), and EurC (broadly the rest of the former USSR and former satellites). The environmental risk factors were much stronger or more prevalent in EurB and C than in EurA and consequently the EBD was much higher in these subregions.

Appropriate actions, targeted according to need, should result in substantial gains in childhealth. Environment, health, transport, energy, urban planning, and education sectors will have to be involved as well as governments as a whole. 